\title{
Análise temporal das distribuições de diâmetros e alturas de uma Floresta Estacional Semidecidual na transição Cerrado- Floresta Amazônica, Leste do Mato Grosso, Brasil
}

\author{
Henrique Augusto Mews ${ }^{1 *}$ \\ Beatriz Schwantes Marimon ${ }^{2}$ \\ Leandro Maracahipes ${ }^{2}$ \\ Edmar Almeida de Oliveira ${ }^{2}$

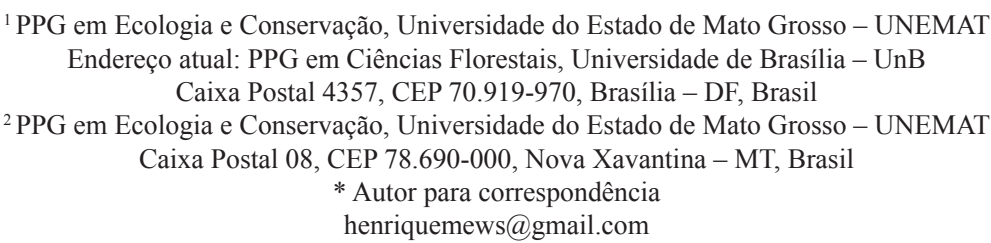

\section{Resumo}

O objetivo deste estudo foi descrever as flutuações temporais nas distribuições de diâmetros e alturas de uma Floresta Estacional Semidecidual (1449'32”S e 5206’20”W), na transição Cerrado-Floresta Amazônica, Nova Xavantina - MT, entre 2003 e 2008. Foram estabelecidas 60 parcelas permanentes de 10 x $10 \mathrm{~m}$, onde foram medidos os diâmetros e as alturas de todos os indivíduos com diâmetro a altura do peito $\geq 5 \mathrm{~cm}$. Os intervalos de classe para as distribuições de diâmetro e altura foram calculados e elaborados histogramas. As distribuições de diâmetro da comunidade e das principais espécies não diferiram no período, bem como a distribuição de alturas da comunidade, caracterizando uma situação de manutenção temporal das distribuições. Apesar da ausência de alterações temporais, a comunidade estudada não é estática, pois entre 2003 e 2008 constatou-se redução do número de indivíduos (6\%) e uma discrepância dos valores do quociente "q" entre as sucessivas classes diamétricas. As distribuições diamétricas das principais espécies sugerem que Cheiloclinium cognatum, Tetragastris altissima e Brosimum rubescens manterão ou eventualmente aumentarão suas densidades populacionais, enquanto Amaioua guianensis e Chaetocarpus echinocarpus possivelmente terão suas populações reduzidas no futuro.

Palavras-chave: Alterações estruturais; Comunidade lenhosa; Dinâmica temporal; Florestas tropicais

\section{Abstract}

Temporal analysis of diameter and height distributions in a Semideciduous Seasonal Forest in the Cerrado-Amazon Forest transition, East Mato Grosso, Brazil. The aim of this study was to describe the temporal fluctuations in diameter and height distributions in a semideciduous seasonal forest (14\%49'32"S and 52 06’20”W), in the Cerrado-Amazon Forest transition, Nova Xavantina - MT, Brazil, between 2003 and 2008. 
Sixty permanent plots of $10 \times 10 \mathrm{~m}$ were established, where the diameters and heights of all individuals with diameter at breast height $\geq 5 \mathrm{~cm}$ were measured. The class intervals for diameter and height distribution were calculated and histograms were constructed. The diameter distributions of the community and the main species did not differ within the period, as well as the community height distribution, characterizing a temporal maintenance of the distributions. Even with the absence of temporal changes, the community under study is not static, since between 2003 and 2008 it was found a decrease in the number of individuals (6\%) and a discrepancy in the "q" quotient values among the successive diameter classes. The diameter distributions of the main species suggest that Cheiloclinium cognatum, Tetragastris altissima and Brosimum rubescens will keep or perhaps increase their population densities, whereas Amaioua guianensis and Chaetocarpus echinocarpus possibly shall have a decrease in their populations in the future.

Key words: Structural changes; Temporal dynamics; Tropical forests; Woody community

\section{Introdução}

Muitos estudos são conduzidos em florestas tropicais e, de forma geral, a maioria é realizada nas florestas úmidas, com menor atenção às sazonalmente secas (MOONEY et al., 1995). No Brasil, dentre os estudos desenvolvidos em florestas sazonalmente secas, vários têm considerado a distribuição das árvores em classes de diâmetros e alturas em suas análises estruturais (OLIVEIRA-FILHO et al., 1994; MARIMON; FELFILI, 2000; ANDRADE; RODAL, 2004; PAULA et al., 2004).

Nos ecossistemas tropicais, a medição dos diâmetros dos troncos permite uma estimativa da estrutura de tamanho das populações, a partir das quais é possível realizar predições sobre a produção dos recursos florestais (HARPER, 1977). Além disso, a distribuição dos diâmetros em classes pode auxiliar no processo de compreensão da sucessão ecológica (PAULA et al., 2004) e organização do componente arbóreo de uma floresta (MARIMON; FELFILI, 2000). A medição dos diâmetros e alturas das árvores pode, muitas vezes, retratar os acontecimentos pelos quais uma determinada floresta passou, além de permitir inferências sobre o seu futuro (FELFILI; SILVA JÚNIOR, 1988; SILVA JÚNIOR; SILVA, 1988; MACHADO et al., 2010). De acordo com Schiavini et al. (2001), as perturbações sofridas ficam impressas na estrutura das populações e, consequentemente, são refletidas na distribuição de diâmetros e alturas da comunidade. Assim, este parâmetro é considerado relevante na compreensão da dinâmica florestal (FELFILI, 1997).

Nas frágeis e ameaçadas florestas estacionais do leste do estado de Mato Grosso, na área de contato entre os Biomas Cerrado e Floresta Amazônica, poucos estudos têm sido conduzidos (MARIMON et al., 2001a; 2001b). Além disso, nesta região os estudos estão relacionados aos aspectos florísticos e estruturais das comunidades (RATTER et al., 1973; IVANAUSKAS et al., 2004; MARIMON et al., 2006) e à ecologia do fogo (BALCH et al., 2008), frequentemente desconsiderando as mudanças ocorridas ao longo do tempo. É importante salientar que tais florestas estão localizadas na região conhecida como o "arco do desmatamento" (NOGUEIRA et al., 2008) e estão sendo drasticamente reduzidas pelo avanço da ocupação antrópica, sendo atualmente um dos tipos florestais mais ameaçados do estado (ALENCAR et al., 2004).

Nesse sentido, o objetivo deste estudo foi avaliar e descrever as alterações ocorridas em um período de cinco anos nas distribuições de diâmetros e de alturas da comunidade e de diâmetros das principais espécies lenhosas de uma Floresta Estacional Semidecidual na zona de transição entre os Biomas Cerrado e Floresta Amazônica no estado de Mato Grosso, visando orientar medidas para seu manejo e conservação.

\section{Material e Métodos}

O estudo foi realizado em uma Floresta Estacional Semidecidual localizada na Fazenda Vera Cruz, Retiro

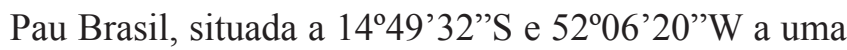
altitude de $250 \mathrm{~m}$. O trecho de floresta selecionado para o estudo é bem preservado, não apresenta vestígios de extração de madeira ou entrada de fogo e compreende parte da reserva legal da fazenda, que ao todo ocupa uma área contínua de 5.000 hectares (MARIMON, 2005). 
O clima predominante na região é do tipo $A w$, de acordo com o sistema de classificação de Köppen, com estações seca e chuvosa bem definidas, inverno seco e chuvas máximas de verão (SILVA et al., 2008), com precipitação média anual de $1.500 \mathrm{~mm}$ (MARIMON et al., 2001b). O relevo da área estudada é plano e o solo é do tipo Latossolo Vermelho-Amarelo Distrófico, bem drenado e com textura média (MARIMON et al., 2001a; MARIMON, 2005). A cobertura vegetal da região do Médio Araguaia mato-grossense se caracteriza pela ocorrência de um mosaico vegetacional composto por formações savânicas e por florestas de transição da préAmazônia (RATTER et al., 1973; IVANAUSKAS et al., 2004). A área estudada é circundada por um mosaico de formações florestais, entre as quais se destacam a floresta monodominante de Brosimum rubescens Taub., a mata de galeria pantanosa e o cerradão (MARIMON et al., 2001a; MARIMON, 2005).

Para a execução deste estudo foram estabelecidas 60 parcelas permanentes de $10 \times 10 \mathrm{~m}$ onde foram amostrados todos os indivíduos vivos e mortos em pé que apresentaram diâmetro a $1,3 \mathrm{~m}$ de altura do solo (DAP) $\geq 5 \mathrm{~cm}$. Na ocasião do primeiro inventário, em 2003, todos os indivíduos receberam placas de alumínio numeradas, foram identificados e tiveram a altura total $\mathrm{e}$ o DAP medidos. Para a medição das alturas foi utilizada uma régua graduada de $12 \mathrm{~m}$, sendo as alturas superiores estimadas (MARIMON, 2005). Em 2008, as parcelas foram reavaliadas, os indivíduos foram remedidos e os recrutas (indivíduos que atingiram o critério mínimo de inclusão) foram incluídos. Os DAP's de indivíduos que apresentavam ramificações desde a base foram determinados empregando-se o cálculo do diâmetro quadrático recomendado por Scolforo (1993).

Foram calculadas, para ambos os inventários, as distribuições dos indivíduos e da área basal viva da comunidade e das principais espécies em classes de diâmetro. Foram consideradas como espécies principais as cinco primeiras em valor de importância - VI com 20 ou mais indivíduos. Juntas, tais espécies representaram aproximadamente $43 \%$ dos indivíduos registrados em ambos os inventários (MEWS, 2010). As espécies foram: Cheiloclinium cognatum (Miers) A.C. Sm. Celastraceae; Amaioua guianensis Aubl. - Rubiaceae;
Tetragastris altissima (Aubl.) Swart - Burseraceae; Chaetocarpus echinocarpus (Baill.) Ducke - Peraceae e Brosimum rubescens Taub. - Moraceae. Adicionalmente, foram calculadas, para ambos os inventários, as distribuições dos indivíduos vivos da comunidade em classes de altura e dos indivíduos e da área basal morta da comunidade em classes de diâmetro. A separação do espaço vertical da floresta em estratos inferior, intermediário e superior foi baseada na classificação proposta por Felfili (1994).

Os intervalos de classe para as distribuições de diâmetros e de alturas foram determinados a partir de Bonini e Bonini (1972), por meio da fórmula: $\mathrm{A} / \mathrm{K}$, onde $A$ representa a amplitude dos valores de diâmetro e $K$ representa uma constante definida pelo algoritmo de Sturges, cuja fórmula é: $1+3,3 \times \log 10$, onde $n$ é o número total de indivíduos amostrados. Para avaliar se houve diferença nas distribuições dos diâmetros e das alturas entre 2003 e 2008 foi aplicado o teste de Kolmogorov-Smirnov (KS) (SIEGEL; CASTELLANJÚNIOR, 1988). Para verificar se o número de indivíduos mortos e área basal morta foram independentes das classes de diâmetro foram aplicadas Regressões Lineares Simples (ZAR, 1999). O cálculos foram realizados por meio do programa BioEstat 5.0 (AYRES et al., 2007).

O quociente " $q$ " de Liocourt foi utilizado para avaliar o balanceamento das distribuições dos diâmetros da comunidade e das principais espécies nos dois inventários. Este parâmetro é um modelo baseado em uma progressão geométrica que estabelece que a razão entre o número de indivíduos em sucessivas classes de diâmetro seria constante em florestas naturais balanceadas, pois nessas florestas o recrutamento seria compensado pela mortalidade (LEAK, 1964).

\section{Resultados e Discussão}

\section{Distribuição de diâmetros da comunidade}

A distribuição dos indivíduos vivos da comunidade em classes de diâmetro, em ambos os inventários (2003 e 2008), apresentou poucos indivíduos de grande porte e muitos indivíduos menores (cerca de $60 \%$ na primeira 
classe) (Figura 1). Este padrão de distribuição sugere que a comunidade é constituída por muitos indivíduos de pequeno porte (HARPER, 1977) ou em fase inicial de estabelecimento, apontando muitas vezes um grande potencial de autorregeneração (SILVA JÚNIOR; SILVA, 1988) e um recrutamento contínuo (WEEB et al., 1972). A distribuição da área basal nas diferentes classes de diâmetro apresentou maiores valores nas classes iniciais em ambos os inventários (Figura 1). Certamente este padrão está relacionado ao elevado número de indivíduos jovens ou de pequeno porte, principalmente de Cheiloclinium cognatum, que somados superam a área basal dos indivíduos das classes maiores.

A distribuição diamétrica apresentou classes ininterruptas e com números similares de indivíduos em ambos os inventários, resultando em pequenas mudanças na densidade de cada classe (Figura 1). Tal condição pode indicar que o ciclo de vida da maioria das espécies está se completando (FELFILI; SILVA JÚNIOR, 1988), fato geralmente relacionado com a ausência de perturbações ambientais, como é o caso da floresta estudada (MARIMON, 2005).
A distribuição de diâmetros da comunidade não diferiu no período de cinco anos, tanto para o número de indivíduos (KS, $\left.\chi^{2}=0,843, p>0,05\right)$ quanto para a área basal (KS, $\chi^{2}=0,034, p>0,05$ ), apesar do número total de indivíduos da comunidade $(684$ em 2003 e 643 em 2008) ter passado por uma redução de $6 \%$ entre os inventários (MEWS, 2010). A ausência de alterações temporais na distribuição diamétrica demonstra que a floresta manteve o mesmo padrão de distribuição registrado no primeiro inventário. No entanto, este fato não deve ser interpretado como uma condição de estagnação estrutural da comunidade, pois a estrutura de uma floresta não é estática, sendo determinada por variações nas condições ambientais e/ou em resposta aos distúrbios naturais (PINTO; HAY, 2005), como a abertura de clareiras resultante da queda de árvores (PINTO; OLIVEIRA-FILHO, 1999; MARIMON, 2005; MARIMON; FELFILI, 2006). No presente estudo, a redução no número de indivíduos entre os inventários, mesmo que não significativa, pode ser um indicativo destas condições.

FIGURA 1: Distribuição diamétrica dos indivíduos vivos da comunidade lenhosa da Floresta Estacional Semidecidual amostrada em Nova Xavantina - MT em 2003 (MARIMON, 2005) e em 2008, considerando o número de indivíduos $(\square=2003$ e $\mathbf{\square}=2008)$ e a área basal $\left(-0_{-}=2003\right.$ e $\left.-\mathbf{m -}=2008\right)$. Valores sobre as barras representam a porcentagem de espécies.

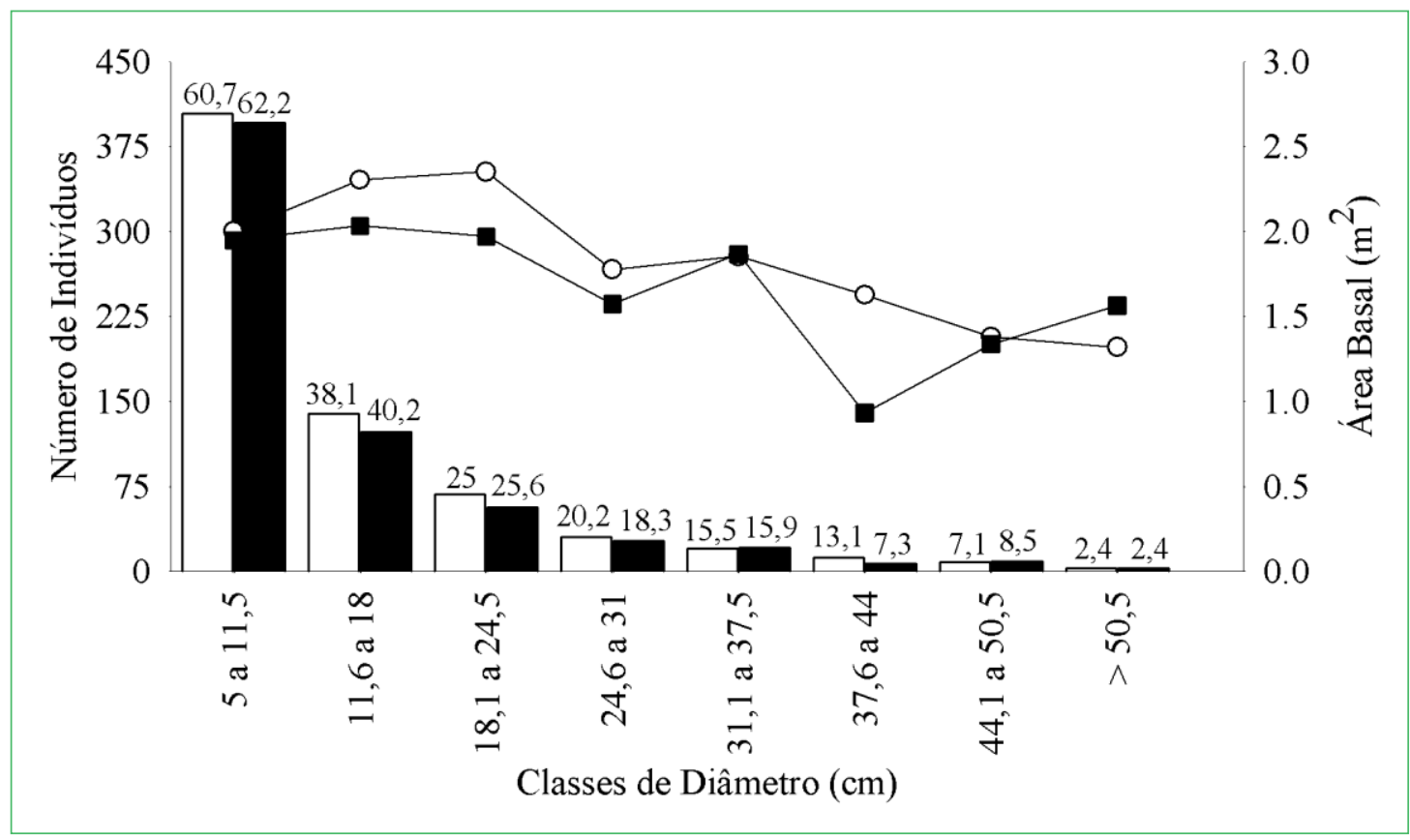


Outro resultado que confirma que a comunidade estudada não é estruturalmente estática, apesar da ausência de alterações significativas nas distribuições de diâmetros, é o fato de os valores do quociente "q" terem variado entre as sucessivas classes diamétricas (Tabela 1). Tal resultado aponta um desbalanceamento das distribuições de ambos os inventários e corrobora o padrão de desbalanceamento descrito por Harper (1977) para florestas tropicais heterogêneas. No entanto, a pequena discrepância entre os valores do quociente "q" nas menores classes sugere uma tendência de constância ou de balanceamento (FELFILI; SILVA JÚNIOR, 1988; FELFILI, 1997; MARIMON et al., 2001b; IVANAUSKAS et al., 2004), enquanto a evidente variação nos valores deste parâmetro nas maiores classes pode ser atribuída, principalmente, ao reduzido número de indivíduos de grande porte (LEAK, 1964).

O número de indivíduos mortos em pé aumentou de 33 no primeiro inventário para 95 no segundo (Figura 2). Embora a inclusão de árvores mortas venha sendo objeto de críticas, por pertencerem a várias espécies, sendo a identificação praticamente impossível, Durigan (2009) destaca que podem ser consideradas em comunidades monitoradas em longo prazo, quando morrerem durante o período de análise, como foi o caso do presente estudo. A mortalidade de indivíduos entre 2003 e 2008 apresentou relação negativa com as classes diamétricas $\left(\mathrm{r}^{2}=0,662 ; \mathrm{F}=11,770 ; p=0,0140\right)$, sendo mais elevada nas classes iniciais e diminuindo com o aumento do diâmetro. Este padrão também foi constatado por
Oliveira-Filho et al. (1994) e Marimon e Felfili (2000) em uma Floresta Estacional Semidecidual em Minas Gerais e uma floresta monodominante em Mato Grosso, respectivamente. Uma possível explicação para tal fenômeno é a maior suscetibilidade dos indivíduos menores aos efeitos da competição, uma vez que na floresta estudada a densidade de indivíduos nas menores classes diamétricas foi elevada e diminuiu com o aumento do diâmetro (Figura 1). Outro fator que pode ter contribuído para a elevada mortalidade de indivíduos de menor porte é o maior sombreamento a que os mesmos normalmente estão sujeitos, como também observaram Oliveira-Filho et al. (1997). Neste caso, espécies que exigem grande incidência de luz nos estádios iniciais de desenvolvimento podem ter sido desfavorecidas, o que também pode ter contribuído para o padrão encontrado na floresta estudada.

A distribuição da área basal morta não apresentou relação significativa com as classes diamétricas $\left(\mathrm{r}^{2}=0,333 ; \mathrm{F}=2,998 ; p=0,1323\right)$, visto que esta foi mais elevada nas primeiras e nas últimas classes. Neste caso, o elevado número de indivíduos jovens ou de pequeno porte que morreu entre os inventários incrementou a área basal morta nas classes iniciais, enquanto nas maiores classes a área basal morta está relacionada à elevada biomassa de alguns poucos indivíduos de grande porte que morreram entre 2003 e 2008 (Figura 2).

Diferentemente do que foi registrado para os indivíduos mortos, o número e a área basal dos

TABELA 1: Quociente “q” calculado para a comunidade e para as principais espécies da Floresta Estacional Semidecidual amostrada em Nova Xavantina - MT em 2003 (T1) (MARIMON, 2005) e em 2008 (T2).

\begin{tabular}{|c|c|c|c|c|c|c|c|c|c|c|c|c|c|c|c|c|}
\hline & \multicolumn{2}{|c|}{ q1 } & \multicolumn{2}{|c|}{ q2 } & \multicolumn{2}{|c|}{ q3 } & \multicolumn{2}{|c|}{$\mathrm{q} 4$} & \multicolumn{2}{|c|}{ q5 } & \multicolumn{2}{|c|}{ q6 } & \multicolumn{2}{|c|}{$\mathrm{q} 7$} & \multicolumn{2}{|c|}{$\mathrm{qm}$} \\
\hline & $\mathrm{T} 1$ & $\mathrm{~T} 2$ & $\mathrm{~T} 1$ & $\mathrm{~T} 2$ & $\mathrm{~T} 1$ & $\mathrm{~T} 2$ & $\mathrm{~T} 1$ & $\mathrm{~T} 2$ & $\mathrm{~T} 1$ & $\mathrm{~T} 2$ & $\mathrm{~T} 1$ & $\mathrm{~T} 2$ & $\mathrm{~T} 1$ & $\mathrm{~T} 2$ & $\mathrm{~T} 1$ & $\mathrm{~T} 2$ \\
\hline $\mathrm{CO}$ & 0,34 & 0,31 & 0,48 & 0,46 & 0,44 & 0,47 & 0,66 & 0,77 & 0,60 & 0,33 & 0,66 & 1,28 & 0,37 & 0,33 & 0,51 & 0,56 \\
\hline $\mathrm{Cc}$ & 0,90 & 111 & 0,72 & & & 0,81 & 0,30 & & & & 0,21 & 0,50 & 0,66 & 0,66 & 0,80 & 0,71 \\
\hline $\mathrm{Ag}$ & 1,69 & 1,46 & 0,63 & & & 0,91 & 0,81 & & 0,55 & & 0,40 & 0,50 & - & - & 0,81 & 0,78 \\
\hline $\mathrm{Ta}$ & 0,60 & 0,75 & 1,66 & 1,50 & 0,30 & 0,11 & 2,66 & 8,00 & 0,25 & 0,25 & - & - & - & - & 1,09 & 2,12 \\
\hline $\mathrm{Ce}$ & 0,75 & 0,40 & 1,66 & 2,50 & 1,00 & 1,00 & 1,40 & 1,20 & 0,42 & 0,66 & - & - & - & - & 1,04 & 1,15 \\
\hline $\mathrm{Br}$ & 0,20 & 0,21 & 1,00 & 0,66 & 0,66 & 1,50 & 0,50 & - & 2,00 & - & - & - & - & - & 0,87 & 0,47 \\
\hline
\end{tabular}

$\mathrm{q}_{\mathrm{m}}=$ valor médio do quociente "q", $\mathrm{CO}=$ Comunidade, $\mathrm{Cc}=$ Cheiloclinium cognatum, Ag = Amaioua guianensis, Ta $=$ Tetragastris altissima, $\mathrm{Ce}=$ Chaetocarpus echinocarpus e $\mathrm{Br}=$ Brosimum rubescens. 
FIGURA 2: Distribuição diamétrica dos indivíduos mortos da comunidade lenhosa da Floresta Estacional Semidecidual amostrada em Nova Xavantina - MT em 2003 (MARIMON, 2005) e em 2008, considerando o número de indivíduos $(\square=2003$ e $\square=2008)$ e a área basal (-O- = 2003 e -m- = 2008).

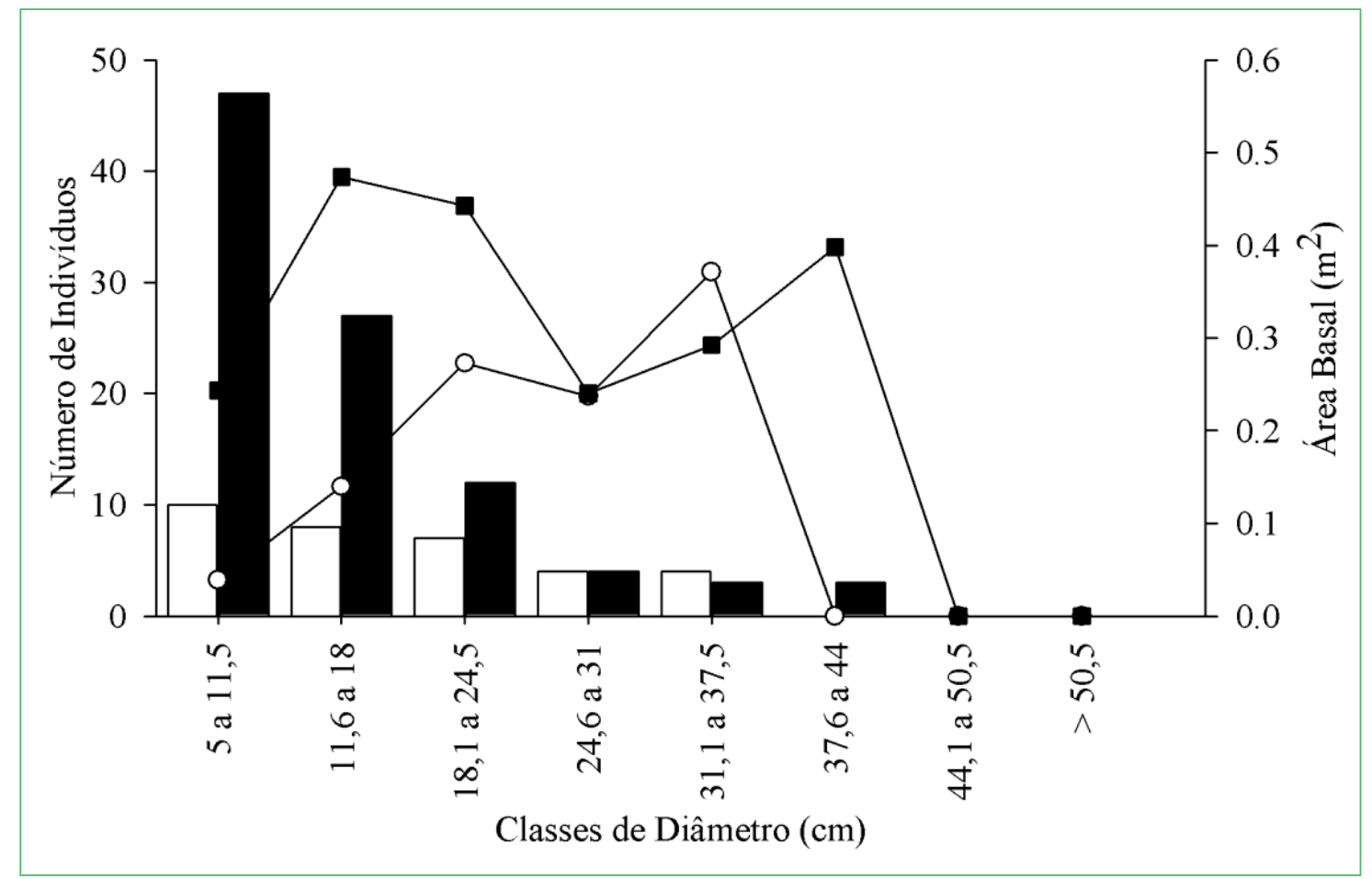

indivíduos vivos praticamente não sofreram alteração entre os dois inventários (Figura 1). Neste caso, o aumento no número de mortos registrados no segundo inventário (Figura 2) pode ser compensado também por recrutas (indivíduos que não haviam atingido o critério mínimo de inclusão no primeiro inventário) que ingressaram nas três primeiras classes e que morreram antes do segundo inventário, reforçando o aspecto da maior vulnerabilidade dos indivíduos jovens.

\section{Distribuição de diâmetros principais espécies}

As cinco espécies aqui estudadas apresentaram padrões distintos de distribuição dos diâmetros e em nenhuma o padrão J-reverso foi constatado (Figura 3). Neste caso, é possível que as espécies analisadas tenham apresentado particularidades nos padrões de distribuição em resposta às suas diferentes estratégias frente à dinâmica dos estratos da floresta, como também salientado por Oliveira-Filho et al. (1994) e Carvalho et al. (1995).
Cheiloclinium cognatum apresentou, nos dois inventários, elevado número de indivíduos nas duas primeiras classes com queda gradativa nas classes seguintes (Figura 3), sendo que as distribuições diamétricas não diferiram no período $\left(\mathrm{KS}, \chi^{2}=0,483\right.$, $p>0,05)$. A área basal foi maior nas maiores classes e não diferiu entre os inventários (KS, $\left.\chi^{2}=0,007, p>0,05\right)$. Neste caso, as distribuições de $C$. cognatum podem estar relacionadas à sua estratégia de vida, pois espécies secundárias tardias tolerantes à sombra, seguindo os critérios sugeridos por Gandolfi et al. (1995), completam o seu ciclo de vida no sub-dossel da floresta e por tal motivo a maioria dos indivíduos pode apresentar pequeno porte (CARVALHO et al., 1995).

Amaioua guianensis apresentou distribuição diamétrica com o maior número de indivíduos na segunda classe, entre 8,6 e 12cm de diâmetro (Figura 3 ), com baixo recrutamento no período (poucos indivíduos na primeira classe) que sugere problemas com a regeneração natural da espécie. Apesar disso, as distribuições diamétricas dos indivíduos (KS, $\chi^{2}=0,058$, 
$p>0,05)$ e da área basal (KS, $\left.\chi^{2}=0,002, p>0,05\right)$ não diferiram entre os inventários. Marimon (2005) estudou a população de $A$. guianensis em uma floresta adjacente durante período anterior e relacionou este padrão a uma população madura e desbalanceada, onde o recrutamento não compensou a mortalidade durante certo período, tal como foi observado no presente estudo, onde a espécie perdeu dez e recrutou apenas dois indivíduos. Em longo prazo, se o padrão de distribuição observado for mantido, a permanência da referida espécie na floresta pode ser comprometida, pois poucos são os indivíduos jovens que irão repor os senis, como observaram Carvalho e Nascimento (2009) em um trecho de Floresta Atlântica submontana no Rio de Janeiro.

A distribuição de Tetragastris altissima, em ambos os inventários, caracterizou-se principalmente pela alternância de classes representadas por um elevado e por um reduzido número de indivíduos, sem grandes alterações nas densidades das classes no período (Figura 3) e não diferindo entre 2003 e 2008 (KS,

FIGURA 3: Distribuição diamétrica das principais espécies lenhosas em uma Floresta Estacional Semidecidual, Nova Xavantina - MT, entre 2003 (MARIMON, 2005) e 2008, considerando o número de indivíduos ( $\square=2003$ e $\mathbf{q}=2008)$ e área basal $\left(-0_{-}=2003\right.$ e $-\mathbf{m -}=2008)$.

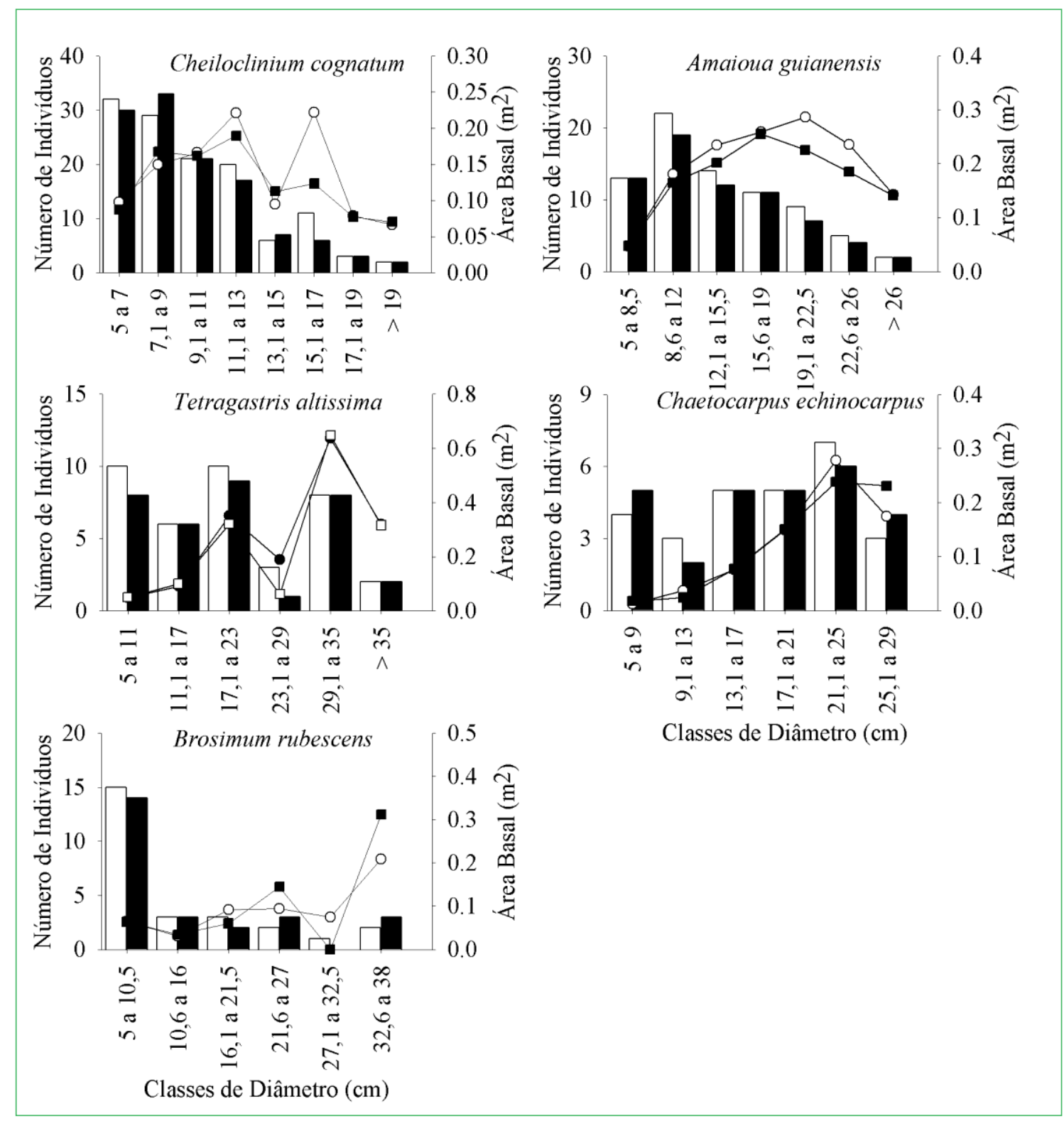


$\left.\chi^{2}=0,103, p>0,05\right)$. A área basal também apresentou padrão de distribuição similar, sem alterações entre os inventários (KS, $\left.\chi^{2}=0,011, p>0,05\right)$. De acordo com Marimon (2005), o fato de a distribuição de T. altissima ter apresentado picos irregulares em algumas classes poderia indicar que algum distúrbio passado afetou os indivíduos das classes anteriores. No entanto, Bongers et al. (1988) e Oliveira-Filho et al. (1994) afirmaram que uma distribuição com picos irregulares entre as classes indicaria que esta espécie apresenta boa reprodução, mas recrutamento descontínuo. Na floresta estudada, $T$. altissima apresenta concentração sazonal de sementes, com curtos períodos de dispersão (MARIMON; FELFILI, 2006), podendo resultar em recrutamento episódico de indivíduos (CLARK; CLARK, 1987). A regularidade com que o número de indivíduos variou nas sucessivas classes sugere que o padrão de recrutamento supracitado é inerente à espécie e ocorre em resposta às suas características reprodutivas.

Em ambos os inventários a distribuição diamétrica de Chaetocarpus echinocarpus apresentou elevada densidade nas classes intermediárias (Figura 3), sugerindo que a espécie apresentou elevado número de indivíduos jovens no passado, provavelmente em função da abertura de clareiras, como observado por Felfili e Silva Júnior (1988). Entretanto, as distribuições de indivíduos em classes de diâmetro não diferiram entre os inventários (KS, $\left.\chi^{2}=0, p>0,05\right)$. Neste caso, é possível que a proporção de indivíduos jovens de $C$. echinocarpus em ambos os inventários tenha sido menor em comparação com períodos anteriores em resposta à redução da incidência de luz no sub-dossel, já que esta se trata de uma espécie secundária tardia do dossel, de acordo com os critérios propostos por Gandolfi et al. (1995), resultando em um menor estabelecimento de indivíduos em relação ao passado. Como reflexo da redução do número de indivíduos jovens, a área basal foi maior nas maiores classes diamétricas, porém não diferindo entre 2003 e 2008 (KS, $\chi^{2}=0,007, p>0,05$ ).

A distribuição de diâmetros de Brosimum rubescens caracterizou-se por apresentar muitos indivíduos na primeira classe e um declínio acentuado nas classes subsequentes (Figura 3), mantendo-se entre os anos de 2003 e 2008 (KS, $\chi^{2}=0,116, p>0,05$ ). Este padrão pode ser reflexo da produção maciça de sementes de B. rubescens nos períodos desfavoráveis para a maioria das espécies, acarretando na formação de um abundante banco de plântulas que são capazes de sobreviver no sub-dossel até encontrarem condições favoráveis de luminosidade para continuar seu crescimento (MARIMON; FELFILI, 2000; 2006). Considerando se tratar de uma espécie secundária tardia típica do dossel, de acordo com os critérios propostos por Gandolfi et al. (1995), em tais condições $B$. rubescens pode apresentar um recrutamento episódico, ou seja, relacionado a episódios de abertura de clareiras (MARIMON; FELFILI, 2000). Tais características inerentes à espécie e associadas à sua estratégia de vida garantiram uma grande concentração de indivíduos jovens, que incrementaram a densidade da primeira classe diamétrica no presente estudo. No entanto, a área basal da referida espécie foi mais elevada nas maiores classes, não diferindo entre 2003 e 2008 (KS, $\chi^{2}=0,022$, $p>0,05)$ e evidenciando o grande acúmulo de biomassa da espécie nos indivíduos de maior porte.

Ao contrário da distribuição de diâmetros observada no presente estudo, Marimon et al. (2001a) registraram padrão tipicamente unimodal para $B$. rubescens em uma floresta monodominante, relacionando tal fato a redução da incidência de luz pelo fechamento do dossel, com consequente redução de indivíduos jovens. Tais resultados reforçam a ideia de que $B$. rubescens apresenta recrutamento episódico de indivíduos e indicam que a floresta estudada apresenta dossel com a presença de clareiras, onde indivíduos jovens de $B$. rubescens ainda estão se estabelecendo e crescendo.

Apesar de não apresentarem alterações significativas nas distribuições de diâmetro entre os dois inventários, as variações nos valores do quociente "q" das principais espécies (Tabela 1) indicam taxas de recrutamento e mortalidade variáveis, refletindo as diferentes estratégias de vida entre essas espécies e o sucesso diferenciado em se estabelecer na comunidade. Assim, a manutenção da estrutura das populações dependerá, entre outros fatores, do sucesso da regeneração natural das mesmas (MARIMON, 2005). De modo geral, os resultados apontam populações desbalanceadas, sugerindo possíveis alterações nos níveis de densidade no futuro. Cheiloclinium cognatum, T. altissima e B. rubescens 
apresentaram distribuições que sugerem uma condição de manutenção ou, possivelmente, de aumento das densidades populacionais, ao passo que $A$. guianensis e C. echinocarpus provavelmente terão, no futuro, suas populações reduzidas na floresta estudada, caso o padrão observado se mantenha.

A manutenção temporal de um elevado número de indivíduos na primeira classe de diâmetros evidenciou que Cheiloclinium cognatum, Tetragastris altissima e Brosimum rubescens apresentam potencial para serem usadas em estudos mais detalhados visando identificar espécies recomendadas para iniciativas de restauração de áreas alteradas de Floresta Estacional Semidecidual na região leste de Mato Grosso. Este padrão pode indicar bom estabelecimento de novos indivíduos e elevada taxa de sobrevivência, características desejáveis para espécies a serem indicadas para o manejo florestal e recuperação de áreas degradadas.

\section{Distribuição de alturas da comunidade}

As alturas da comunidade, considerando os dois inventários, variaram entre 1,7 e $28 \mathrm{~m}$ e a maioria dos indivíduos ficou situada nas classes intermediárias (limite de 15,5m) (Figura 4), caracterizando um padrão de distribuição unimodal. A distribuição dos indivíduos da comunidade em classes de altura corroborou o padrão apontado por Pinto e Oliveira-Filho (1999) e Marimon et al. (2001b) como natural e típico de florestas tropicais heterogêneas que estão se autorregenerando. Na floresta estudada, a abertura de clareiras no dossel pode ter sido um fator determinante para a grande concentração de indivíduos nas classes intermediárias, como verificado por Pinto e Oliveira-Filho (1999). Neste contexto, os resultados sugerem que muitas clareiras foram abertas em um passado recente, pela queda total ou parcial de árvores mortas, resultando em grande concentração de indivíduos nas classes intermediárias no presente.

A redução de $6 \%$ no número total de indivíduos da comunidade entre 2003 e 2008 foi refletida nos estratos inferior e superior, com percentuais de perda em torno de $11 \%$ para ambos. Neste caso, a redução da densidade de indivíduos do estrato inferior da floresta reforça novamente a maior vulnerabilidade e consequente mortalidade dos indivíduos de menor porte, enquanto a redução em densidade do estrato superior pode estar relacionada à mortalidade de indivíduos de maior porte, que estavam mais senis e com o seu ciclo de

FIGURA 4: Distribuição das alturas da comunidade lenhosa $(\square=2003$ e $\mathbf{~}=2008)$ da Floresta Estacional Semidecidual amostrada em Nova Xavantina - MT.

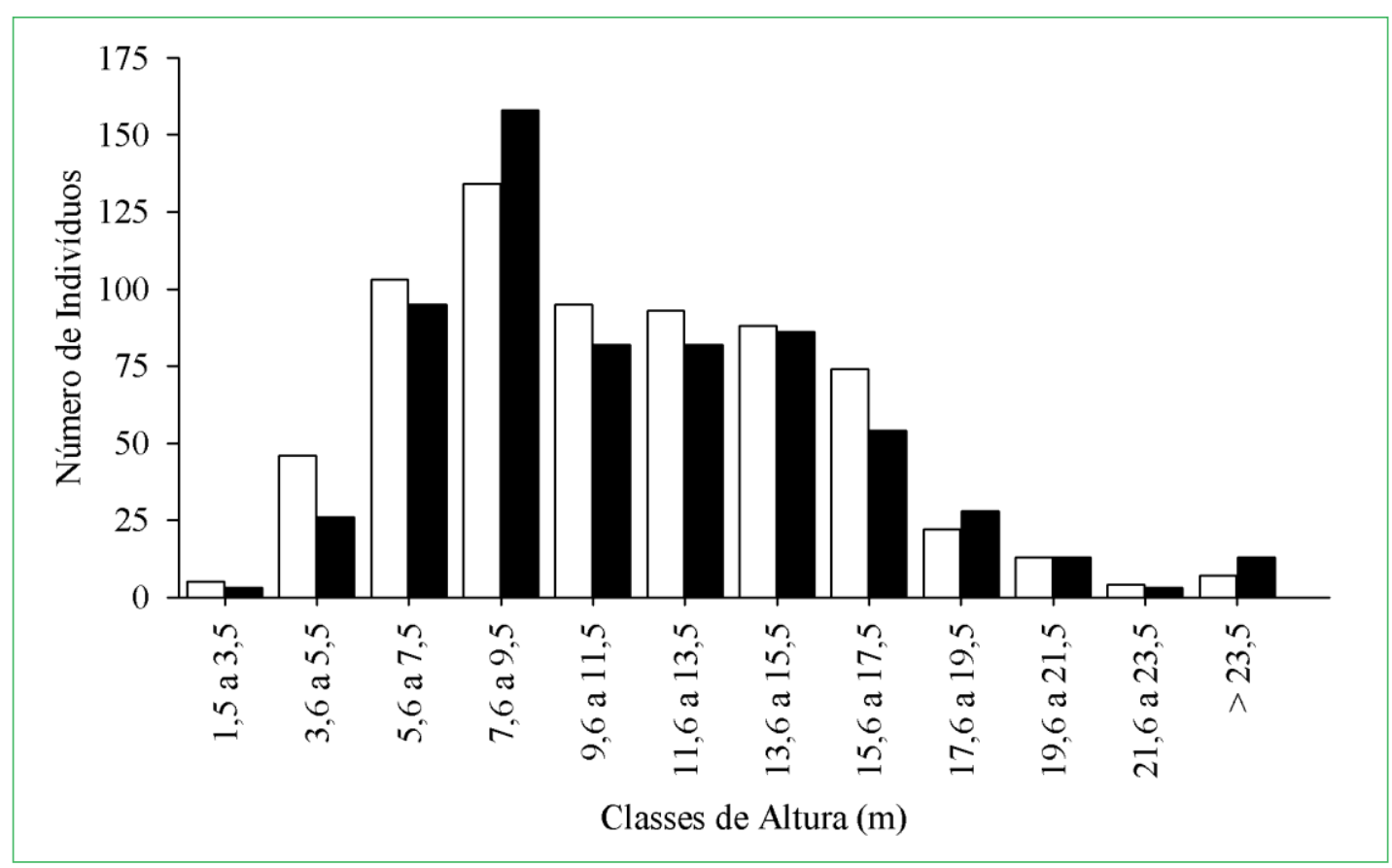


vida sendo encerrado. $\mathrm{O}$ estrato intermediário manteve o mesmo número de indivíduos no período e o grupo dos emergentes aumentou em aproximadamente $23 \%$. Apesar disso, a distribuição de alturas não diferiu entre os inventários (KS, $\left.\chi^{2}=1,3831, p>0,05\right)$ e as mudanças na densidade de cada classe foram pequenas (Figura 4).

Tal como verificado com os diâmetros, a manutenção da distribuição das alturas da comunidade no período estudado indica uma floresta autossustentável, com pequenas alterações ocorrendo de forma muito lenta. No entanto, cabe salientar a importância de se interpretar tais padrões com cautela devido ao fato das alturas superiores a $12 \mathrm{~m}$ terem sido estimadas. Ressalta-se a necessidade de constante monitoramento da floresta com o objetivo de fornecer informações que aumentem o entendimento da dinâmica e contribuam para ações de conservação, manejo e restauração de áreas que tenham sido degradadas.

\section{Agradecimentos}

Ao Sr. Jairo Machado por autorizar a realização do estudo na Fazenda Vera Cruz. Aos professores Dr. Eddie Lenza de Oliveira e Dr. Fernando Pedroni pela leitura crítica do manuscrito. Aos biólogos Pábio H. Porto e Claudinei O. dos Santos pela ajuda nos trabalhos de campo. À CAPES pela concessão de bolsa de estudos à H. A. Mews, L. Maracahipes e E. A. de Oliveira.

\section{Referências}

ALENCAR, A.; NEPSTAD, D.; MCGRATH, D.; MOUTINHO, P.; PACHECO, P.; DIAZ, M. C. V.; SOARES FILHO, B. Desmatamento na Amazônia: indo além da "emergência crônica". Belém: IPAM, 2004. 85 p.

ANDRADE, K. V. S. A.; RODAL, M. J. N. Fisionomia e estrutura de um remanescente de floresta estacional semidecidual de terras baixas no nordeste do Brasil. Revista Brasileira de Botânica, São Paulo, v. 27, n. 3, p. 463-474, 2004.

AYRES, M.; AYRES JR. M.; AYRES, D. L.; SANTOS, A. A. S. BioEstat 5.0: aplicações estatísticas na área das ciências biomédicas. Belém: Sociedade Civil de Mamirauá, 2007. 364 p.

BALCH, J. K.; NEPSTAD, D. C.; BRANDO, P. M.; CURRAN, L. M.; PORTELA, O.; CARVALHO JR., O.; LEFEBVRE, P. Negative fire feedback in a transitional forest of southeastern Amazonia. Global Changes Biology, Oxford, v. 14, p. 1-12, 2008.
BONGERS, F.; POPMA, J.; MEAVE DEL CASTILLO, J.; CARABIAS, J. Structure and floristic composition of the lowland rain forest of Los Tuxtlas, México. Vegetatio, Perth, v. 74, p. 55-80, 1988.

BONINI, E. E.; BONINI, S. E. Estatística teórica e exercícios. São Paulo: Loyola, 1972. 100 p.

CARVALHO, D. A.; VILELA, E. A.; OLIVEIRA-FILHO, A. T.; GAVILANES, M. L. Estrutura diamétrica e vertical de uma floresta ripária no alto Rio Grande (Bom Sucesso - estado de Minas Gerais). Revista Árvore, Viçosa, v. 19, n. 4, p. 572-586, 1995.

CARVALHO, F. A.; NASCIMENTO, M. T. Estrutura diamétrica da comunidade e das principais populações arbóreas de um remanescente de floresta atlântica submontana (Silva Jardim-RJ, Brasil). Revista Árvore, Viçosa, v. 33, n. 2, p. 327-337, 2009.

CLARK, D. A.; CLARK, D. B. Análisis de la regeneración de árboles del dosel em bosque muy húmedo tropical: aspectos teóricos y práticos. Revista de Biología Tropical, San José, v. 35, supl. 1, p. 41-54, 1987.

DURIGAN, G. Estrutura e diversidade de comunidades florestais. In: MARTINS, S. V. (Ed.). Ecologia de florestas tropicais do Brasil. Viçosa: Editora UFV, 2009. p. 185-215.

FELFILI, J. M. Floristic composition and phytosociology of the gallery forest alongside the Gama stream in Brasília, DF, Brazil. Revista Brasileira de Botânica, São Paulo, v. 17, n. 1, p. 1-11, 1994.

FELFILI, J. M. Diameter and height distributions in a gallery forest tree community and some of its main species in central Brazil over a six-year period (1985-1991). Revista Brasileira de Botânica, Feira de Santana, v. 20, n. 2, p. 155-162, 1997.

FELFILI, J. M.; SILVA JÚNIOR, M. C. Distribuição dos diâmetros numa faixa de cerrado na Fazenda Água Limpa (FAL) em BrasíliaDF. Acta Botanica Brasilica, Feira de Santana, v. 2, n. 1-2, p. 85104, 1988.

GANDOLFI, S.; LEITÃO FILHO, H. F.; BEZERRA, C. L. E. Levantamento florístico e caráter sucessional das espécies arbustivo arbóreas de uma floresta mesófila semidecídua no município de Guarulhos, SP. Revista Brasileira de Biologia, São Carlos, v. 55, n. 4, p. 753-767, 1995.

HARPER, J. L. Population biology of plants. London: Academic Press, 1977. $892 \mathrm{p}$.

IVANAUSKAS, N. M.; MONTEIRO, R.; RODRIGUES, R. R. Estrutura de um trecho de floresta Amazônica na bacia do alto Rio Xingu. Acta Amazonica, Manaus, v. 34, n. 2, p. 275-299, 2004.

LEAK, W. B. An expression of diameter distribution for unbalanced uneven-aged stands and forests. Forest Science, Bethesda, v. 10, p. 39-50, 1964.

MACHADO, E. L. M.; GONZAGA, A. P. D.; CARVALHO, W. A. C.; SOUZA, J. S.; HIGUCHI, P.; SANTOS, R. M.; SILVA, A. C.; OLIVEIRA-FILHO, A. T. Flutuações temporais nos padrões de distribuição diamétrica da comunidade arbóreo-arbustivo e de 15 populações em um fragmento florestal. Revista Árvore, Viçosa, v. 34, n. 4, p. 723-732, 2010

MARIMON, B. S. Dinâmica de uma floresta monodominante de Brosimum rubescens Taub. e comparação com uma floresta mista em Nova Xavantina - MT. 2005. 263 f. Tese (Doutorado em Ecologia) - Universidade de Brasília, Brasília. 2005. 
MARIMON, B. S.; FELFILI, J. M. Distribuição de diâmetros e alturas na floresta monodominante de Brosimum rubescens Taub. na Reserva Indígena Areões, Água Boa - MT, Brasil. Revista Árvore, Viçosa, v. 24, n. 2, p. 143-150, 2000.

MARIMON, B. S.; FELFILI, J. M. Chuva de sementes em uma floresta monodominante de Brosimum rubescens Taub. e em uma floresta mista adjacente no Vale do Araguaia, MT, Brasil. Acta Botanica Brasilica, Feira de Santana, v. 20, n. 2, p. 423-432, 2006.

MARIMON, B. S., FELFILI, J. M.; HARIDASAN, M. Studies in monodominant forests in eastern Mato Grosso, Brazil: I. A forest of Brosimum rubescens Taub. Edinburgh Journal of Botany, Edinburgh, v. 58, n. 1, p. 123-137, 2001a.

MARIMON, B. S. FELFILI, J. M.; LIMA, E. S.; RODRIGUES, A. J. Distribuições de circunferências e alturas em três porções da Mata de Galeria do Córrego Bacaba, Nova Xavantina - MT. Revista Árvore, Viçosa, v. 25, n. 3, p. 335-343, 2001 b.

MARIMON, B. S.; LIMA, E. S.; DUARTE, T. G.; CHIEREGATTO, L. C.; RATTER, J.A. Observations on the vegetation of northeastern Mato Grosso, Brazil. IV. Na analysis of the Cerrado-Amazonian Forest ecotone. Edinburgh Journal of Botany, Edinburgh, v. 63, n. 2/3, p. 323-341, 2006.

MEWS, H. A. Dinâmica da comunidade lenhosa de uma floresta estacional semidecidual na transição Cerrado-Floresta Amazônica, leste de Mato Grosso, Brasil. 2010. 90 f. Dissertação (Mestrado em Ecologia e Conservação) - Universidade do Estado de Mato Grosso, Nova Xavantina. 2010.

MOONEY, H. A.; BULLOCK, S. H.; MEDINA, E. Introduction. In: BULLOCK, S. H.; MOONEY, H. A.; MEDINA, E. (Eds). Seasonally dry tropical forests. Cambridge: Cambridge University Press, 1995. p. 1-8.

NOGUEIRA, E. M.; NELSON, B. W.; FEARNSIDE, B. W.; FRANÇA, M. B.; OLIVEIRA, A. C. A. Tree height in Brazil's "arc of deforestation": shorter trees in south and southwest Amazonia imply lower biomass. Forest Ecology and Management, Amsterdan, v. 255, p. 2.963-2.972, 2008.

OLIVEIRA-FILHO, A. T.; SCOLFORO, J. R. S.; MELLO, J. M. Composição florística e estrutura comunitária de um remanescente de floresta semidecídua montana em Lavras, MG. Revista Brasileira de Botânica, São Paulo, v. 17, n. 2, p. 167-182, 1994.

OLIVEIRA-FILHO, A. T.; MELLO, J. M.; SCOLFORO, J. R. Effects of past disturbance and edges on tree community structure and dynamics within a fragment of tropical semideciduous forest in south-eastern Brazil over a five-year period (1987-1992). Plant
Ecology, Perth, v. 131, p. 45-66, 1997.

PAUlA, A.; SILVA, A. F.; DE MARCO JR, P.; SANTOS, F. A. M.; SOUZA, A. L. Sucessão ecológica da vegetação arbórea em uma floresta estacional semidecidual em Viçosa, MG, Brasil. Acta Botanica Brasilica, Feira de Santana, v. 18, n. 3, p. 407-423, 2004.

PINTO, J. R. R.; HAY, J. D. V. Mudanças florísticas e estruturais na comunidade arbórea de uma floresta de vale no Parque Nacional da Chapada dos Guimarães, Mato Grosso, Brasil. Revista Brasileira de Botânica, São Paulo, v. 28, n. 3, p. 523-539, 2005.

PINTO, J. R. R.; OLIVEIRA-FILHO, A. T. Perfil florístico e estrutura da comunidade arbórea de uma floresta de vale no Parque Nacional da Chapada dos Guimarães, Mato Grosso, Brasil. Revista Brasileira de Botânica, São Paulo, v. 22, p. 53-67, 1999.

RATTER, J. A.; RICHARDS, P. W.; ARGENT, G.; GIFFORD, D. R. Observations on the vegetation of the northeastern Mato Grosso. I. The Woody vegetation types of the Xavantina-Cachimbo Expedition area. Philosophical Transactions of the Royal Society of London, London, v. 266, p. 449-492, 1973.

SCHIAVINI, I.; RESENDE, J. C. F.; AQUINO, F. G. Dinâmica de populações de espécies arbóreas em Mata de Galeria e Mata Mesófila na margem do Ribeirão Panga, MG. In: RIBEIRO, J. F.; FONSECA, C. E. L. DA; SOUSA-SILVA, J. C. (EdS). Cerrado: caracterização e recuperação de Matas de Galeria. Planaltina: Embrapa Cerrados, 2001. p. 267-299.

SCOLFORO, J. R. S. Mensuração florestal 5: crescimento florestal 1. Lavras: ESAL/FAEPE, 1993. 292 p.

SIEGEL, S.; CASTELLAN-JÚNIOR, N. T. Nonparametric statistics for the behavioral sciences. New York: McGraw-Hill, 1988. 312 p.

SILVA, F. A. M.; ASSAD, E. D.; EVANGElistA, B. A. Caracterização climática do Bioma Cerrado. In: SANO, S. M., ALMEIDA, S. P.; RIBEIRO, J. F. (Eds). Cerrado: ecologia e flora. Brasília: Embrapa Informação Tecnológica, 2008. p. 69-88.

SILVA JÚNIOR, M. C.; SILVA, A. F. Distribuição dos diâmetros dos troncos das espécies mais importantes do cerrado na estação florestal de experimentação de Paraopeba (EFLEX)-MG. Acta Botanica Brasilica, Feira de Santana, v. 2, n. 1-2, p. 107-126, 1988.

ZAR, J. H. Biostatistical analysis. New Jersey: Prentice Hall, 1999. $663 \mathrm{p}$.

WEEB, L. S.; TRACEY, J. G.; WILLIANS, W. T. Regeneration and pattern in the subtropical rain forest. Journal of Ecology, London, v. 60 , p. $675-695,1972$. 